behind surface reconstruction and relaxation, charge transfer, on-surface synthesis and surface catalysis - insights that could also help in the bottom-up design of molecular devices. Furthermore, the analysis of the electric potentials in the tip-surface region provided in this work might also help to elucidate the relationship between surface potentials and forces in KPFM. So far Wagner and colleagues have limited their studies to the (111) crystal face of Ag, which allows the formation of a molecular quantum dot on the tip by the transfer of a PTCDA molecule from the surface to the tip. Thus, it remains to be seen to what extent the SQDM can be realized on other surfaces, or if it would be limited to specific molecules and surfaces that allow tip functionalization by surfacemolecule transfer. Yet functionalizing the tip by other means, for instance by lithographically fabricated quantum dots as proposed by the researchers, may overcome this limitation. Finding alternative strategies to apply a voltage bias to the quantum dot that do not require the substrate to be electrically connected would also allow the use of SQDM to non-metallic surfaces, for instance in the study of bio-surfaces.
Liverpool, Liverpool, UK. ${ }^{2}$ Department of Chemistry, University of Liverpool, Liverpool, UK.

e-mail:mats.persson@liverpool.ac.uk

Published online: 10 June 2019

https://doi.org/10.1038/s41563-019-0383-7

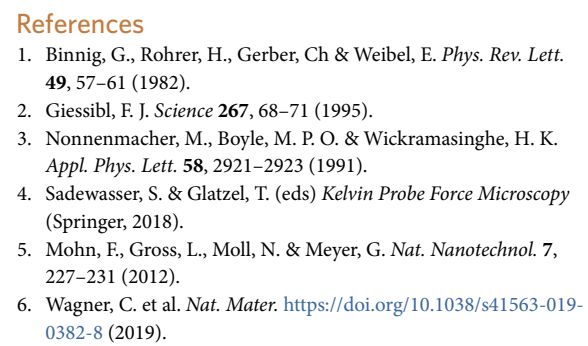

References

Binnig, G., Rohrer, H., Gerber, Ch \& Weibel, E. Phys. Rev. Lett. 49, 57-61 (1982)

3. Nonnenmacher, M., Boyle, M. P. O. \& Wickramasinghe, H. K. Appl. Phys. Lett. 58, 2921-2923 (1991)

4. Sadewasser, S. \& Glatzel, T. (eds) Kelvin Probe Force Microscopy ger, 2018)

227-231 (2012) 0382-8 (2019).

\title{
A new twist on superlubricity
}

\section{L} ubrication has traditionally relied on fluids and other additives to reduce frictional dissipation as one solid surface moves over another. But over the past decade or so it has become clear that dry sliding at the microscale can become almost frictionless when there is incommensurability in the atomicscale structure of the two surfaces ${ }^{1}$. This effect was predicted in the $1980 \mathrm{~s}^{2}$, and dubbed superlubricity in $1991^{3}$ - but only more recently have technological developments in microscale fabrication and manipulation advanced to the point where it can be seen and investigated.

The basis of superlubricity is easily stated. Incommensuration of the atomic-scale corrugations of the surfaces - for example, when a flake of graphite or graphene is rotated relative to the lattice orientation of a graphitic surface on which it rests ${ }^{4}-$ means that there can be almost complete cancellation of the energy barriers to lateral motion: some of the corrugations move 'uphill' while others move 'downhill'. More recently, such almost friction-free motion has been seen at the microscale by using a fine probe tip to shear a tiny flake of graphite from its underlying layers on a microfabricated island (mesa) ${ }^{5}$. The frictional force depended on the orientation of the shearing motion. When the pushing tip was removed, the top flake retracted to its initial position atop the mesa so as to minimize the interfacial energy - something only possible when the frictional forces are so low that this interfacial energy becomes the dominant determinant of the structure's configuration.

Quanshui Zheng of Tsinghua University - one of the authors of that earlier study - and co-workers have now uncovered a new aspect of this phenomenon. They find that a flake pushed in this manner may spontaneously rotate after it has moved a certain distance, in order to move into a lower-energy commensurate relationship with the lower surface ${ }^{6}$. Once the rotation happens and the two surfaces attain a commensurate configuration, the top flake becomes frictionally locked in place.

The rotational instability happens after a predictable amount of (superlubricity-aided) sliding, because it involves a specific balancing of energies. As the flake moves, the contact area decreases, exposing free surface, and thus the total surface energy increases. Still, this configuration remains in a local, metastable state. But at some critical displacement it becomes unstable and rotation takes the system to a stable equilibrium. This happens for a smaller displacement, in general, if the tip pushes from a position displaced from the centreline of the top flake, since that induces a torque that helps to overcome the metastable energy barrier.

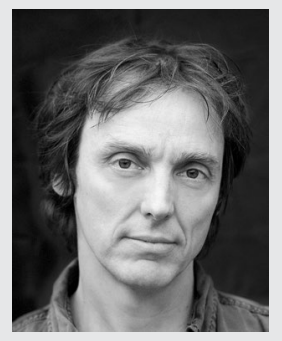

Philip Ball

Understanding this rotational effect in superlubricity should enable it to be controlled, for example so that it doesn't upset efforts to make use of such low-friction interfaces in microscale mechanical devices. Zheng and colleagues have already shown in theoretical calculations that the rotation might be suppressed for a rectangular flake sliding almost friction-free along a 'guide rail' of the same width. Any rotation would decrease the contact area and thus increase the surface energy: here the perfectly aligned sliding state is an energy minimum, so the slider literally stays on track.

Published online: 22 July 2019 https://doi.org/10.1038/s41563-019-0450-0

References

1. Hod, O., Meyer, E., Zheng, Q. \& Urbakh, M. Nature 563, 485-492 (2018).

2. Peyrard, M. \& Aubry, S. J. Phys. C 16, 1593-1608 (1983).

3. Hirano, M., Shinjo, K., Kaneko, R. \& Murata, Y. Phys. Rev. Lett. 67, 2642-2645 (1991).

4. Dienwiebel, M. et al. Phys. Rev. Lett. 92, 126101 (2004).

5. Liu, Z. et al. Phys. Rev. Lett. 108, 205503 (2012).

6. Qu, C., Shi, S., Ma, M. \& Zheng, Q. Phys. Rev. Lett. 122, 246101 (2019). 